It is important to note that dogs with long continued obstruction or closed intestinal loops will survive lethal doses of pure proteose with but few clinical symptoms of intoxication. Dogs recovering from a sterile pleurisy or peritonitis also show a definite resistance or tolerance to subsequent proteose injections. All this evidence strengthens the argument that a proteose intoxication is present in these various conditions.

Other conditions in which inflammation and pus formation or tissue destruction are conspicuous may be considered in which it is possible that toxic proteoses may be concerned. We have made experiments with sterile pleurisy, considered as identical with pertitonitis, and with sterile abscess formation, but do not care to report our findings at this time. Infarcts, pneumonia and many other conditions are interesting possibilities which are being studied.

I feel confident that sufficient evidence has accumulated to show that there is a definite proteose intoxication in intestinal obstruction and allied conditions, in general peritonitis, either septic or sterile, and in acute hemorrhagic pancreatitis. I believe that the proteose intoxication is the most important factor in the general intoxication noted in these conditions. The detailed experiments will be published in the near future.

\title{
THE USES OF THE DESICCATION METHOD IN OPHTHALMOLOGY
}

\section{WITH SPECIAL REFERENCE TO EPITHELIOMAS OF LIDS, CANTHI AND CONJUNCTIVA: REPORT OF CASES *}

WILLIAM L. CLARK, M.D. PHILADELPHIA

The success obtained in some ophthalmologic lesions by the desiccation method is my reason for relating as clearly and briefly as possible what has been achieved up to the present time.

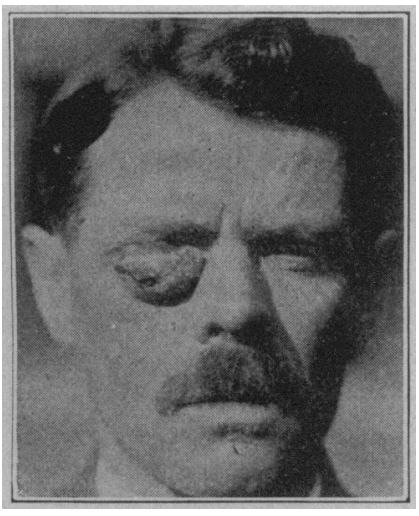

Fig. 1.-Round cell sarcoma of lower lid; condition before excision.

Details concerning this method have already been published. ${ }^{1}$ In the present paper only those points will be emphasized that seem essential for a proper understanding of the application to ophthalmology.

* Read before the Section on Ophthalmology at the Sixty-Seventh Annual Session of the American Medical Association, Detroit, June, 1916 .

1. Clark, W. L.: The Desiccation Treatment of Congenital and New Growths of the Skin and Mucous Membranes, The Journal A. M. A. Sept. 12, 1914, p. 925.

The desiccation method is one by means of which certain congenital or new growths may be reduced by employing heat of just sufficient intensity to desiccate, but not to carbonize them. This heat is best generated by means of a high frequency electric current, which is subject to accurate regulation. I have been unable to produce a current possessing the proper qualities for eye work from any apparatus except a high speed static machine equipped with suitable accessories for transforming the static into a high frequency current.

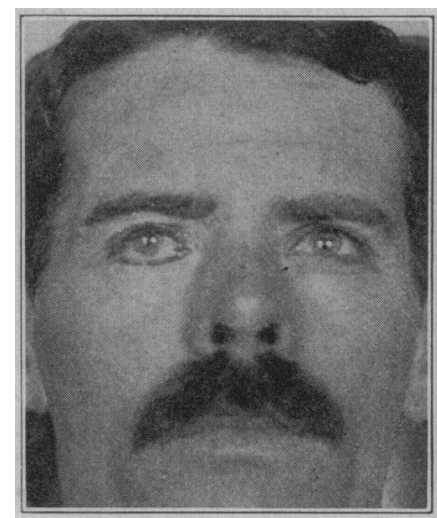

Fig. 3. - Condition one month after one desiccation treatment.

The current thus produced has been found to be smoother and subject to more refined regulation than that generated from an induction coil or transformer. This difference in quality of the static and the coil current differentiates desiccation from destructive fulguration.

The technic for eye applications is as follows: The patient reclines on an operating table which is grounded, if made of metal; otherwise grounding is not necessary. The area desired to be treated is anesthetized locally by the means that seems best for the case under treatment. For the conjunctiva, a 4 per cent. solution of cocain, and for the lids the infiltration of a 1 per cent. solution of novocain and epinephrin are satisfactory. The current is tested so that the desired thermic intensity may be produced. An extremely fine steel needle set in a suitable insulated holder is the best applicator for ophthalmologic growths. It is an advantage to have an assistant make and break the current at given signals, so that the operator may give his whole attention to the work in hand. The needle is not usually thrust into the tissue, but just allowed to brush it. The depth of desiccation depends on the time of contact and the driving power of the current. These can be regulated. An eye speculum or chalazion clamp may be used to expose the lesion better, as may a fixation forceps to keep the eyeball or lids steady. No arbitrary rules can be given for this application, for different cases demand variation in treatment, and a successful technic must be developed by practice.

The desiccation method is peculiarly adaptable to ophthalmologic growths, because of the ease of application and great refinement of control. It is possible to desiccate a minute growth or pigmentation even on the cornea without danger of penetrating to an undesirable depth. Likewise part or whole of one or both eyelids may be removed with precision. Furthermore, cartilage, periosteum or bone in proximity to or com- 
prising the orbit may likewise be devitalized by desiccation, provided these structures are directly accessible.

After a growth has been converted into a dry mass by the treatment, it is usually curetted away or excised immediately after desiccation; but sometimes it is better to allow it to slough. Conjunctival growths become macerated by the lacrimal secretion and slough quickly. There should be no bleeding. A good cosmetic result follows the desiccation treatment, because no sutures are necessary and the cicatrix does not show

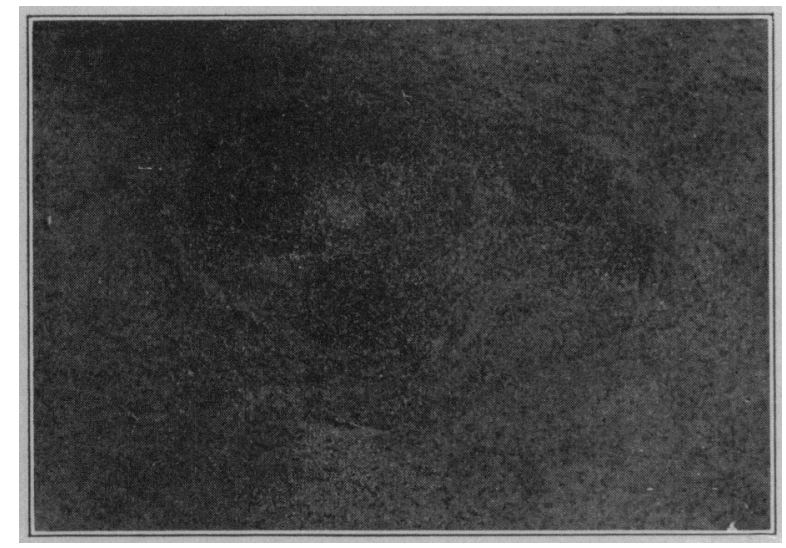

Fig. 5.-Photomicrograph from growth shown in Figure 1.

a tendency to contract as after cautery, electrolysis or, indeed, excision. Infection has never been observed after this treatment, and blood and lymph channels are sealed. A fact worthy of special note is that in case of epitheliomas of the lids, it is not always necessary to desiccate quite all the malignant tissue, as the heat penetration is sufficient to cause a retrogression and final disappearance of the remaining cancer cells, while the normal tissue recovers. On account of this property possessed by the desiccation method, it is often possible to add to the cosmetic result, and to prevent exposure of the eyeball, with its incident dangers.

My experience with the desiccation method in ophthalmologic growths covers a period of seven years, and comprises the lesions mentioned in the accompanying tabulation.

\section{LESIONS TREATED BY AUTHOR}

Epitheliomas of the lids, canthi, conjunctiva and adjacent

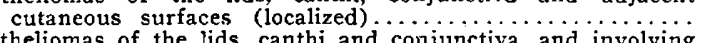
pitheliomas of the lids, canthi and conjunctiva, and involving sinuses or orbit (very advanced).

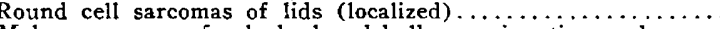
Melanosarcomas of palpebral and bulbar conjunctiva, and cornea Angiomas of lids. . . . . . . . . . . . . . . . . . . . . . Pigmented and other moles, and warts of lids..............

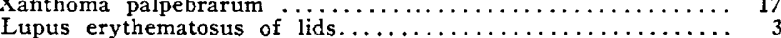

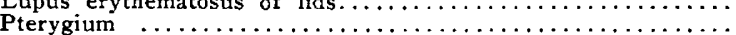

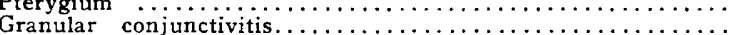

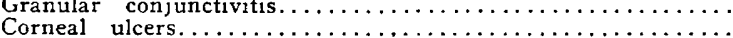
Total number of lesions treated $\ldots \ldots \ldots \ldots \ldots \ldots \overline{186}$

Number 84 8 8 4 7

Epitheliomas of the lids, canthi, conjunctiva and adjacent cutaneous surfaces, as the brow, nose and cheek, are in a large percentage of cases of the basal cell type, and therefore of low grade malignancy and slow of progression, and they seldom metastasize. This is not the case with epitheliomas of the prickle or cuboid cell types which are frequently found in mucous membranes such as the lips, tongue and buccal surfaces as well as in the skin of the extremities, etc. These usually progress rapidly and metastasize early. In the case of basal cell epitheliomas, local attack if thorough should result in permanent relief in practically all cases, while with the prickle or cuboid cell epitheliomas wide ablation and dissection of all adjacent glands is imperative, and even when this is done the results are, as all know, far from satisfactory. Knowing, then, that the great majority of ophthalmologic epitheliomas are of low grade malignancy, it seems a duty to choose, from the various methods of treatment, one that insures a good cosmetic result as well as at least an equal chance of cure.

It has been found that the desiccation method possesses these combined advantages. It is quite true that even the basal cell type may progress to a fatal termination; but this, I believe, is due to long neglect or improper treatment.

I have hitherto refrained from comparing the respective merits of methods employed by various advocates for the treatment of these lesions, but the time has come when I feel justified in doing so.

ADVANTAGES OF THE DESICCATION METHOD

The desiccation method has the following advantages over operative surgery in localized basal cell epitheliomas in ophthalmologic regions:

1. Eradication is at least as thorough, and no sutures are necessary. It is a well known fact that sutures often favor recurrences.

2. There is less chance of recurrence because blood and lymph channels are sealed..

3 . In case of recurrence, which sometimes happens, the lesion may be treated again without materially lessening the chances of ultimate cure or cosmetic result.

4. There are better cosmetic results, because of the absence of contracted cicatrix.

Advantages over radium and the Roentgen rays are:

1. One treatment is usually all that is necessary.

2. There is a better standardization of dosage.

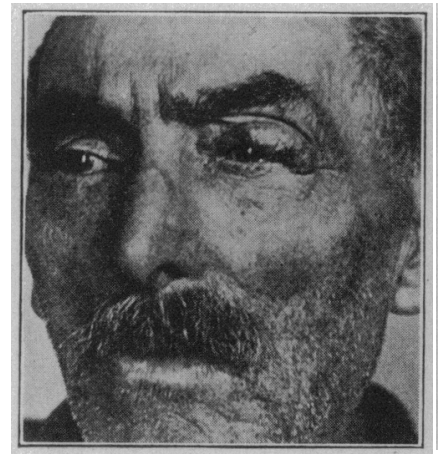

Fig. 6. - Epithelioma of upper lid, involving cartilage.

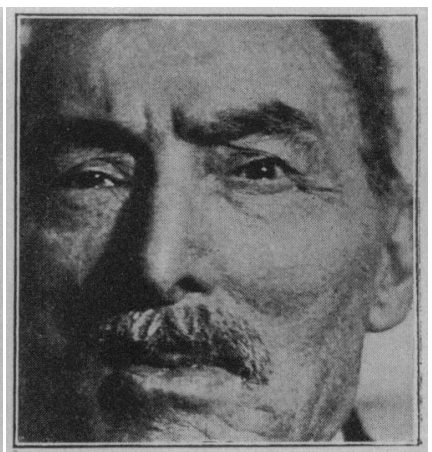

Fig. 7.-Result of one desicca. tion treatment; cartilage conv. served; no recurrence in six months; note absence of con. tracted cicatrix.

3. Action is confined to the area under treatment, and hence there is less danger of impairing the vision or the vitality of adjacent tissues.

4. The effect of desiccation is constant, while that of radium and the Roentgen rays is variable in different cases. It has been observed in every case where the Roentgen rays were used previously without success, that the results have not been as prompt as when desiccation alone had been employed. This seems to be due to impairment of vitality of tissues by the continued use of the Roentgen rays. 
Advantages over the cautery, carbon dioxid snow, liquid air, arsenic or zinc chlorid plasters or other escharotics are:

1. There is devitalization of growth with one application and immediate removal of the growth.

2. There is greater precision of application and certainty of thoroughness.

3. There is less danger of stimulating growths by inadequate destruction.

4. There is conservation of normal surrounding tissue.

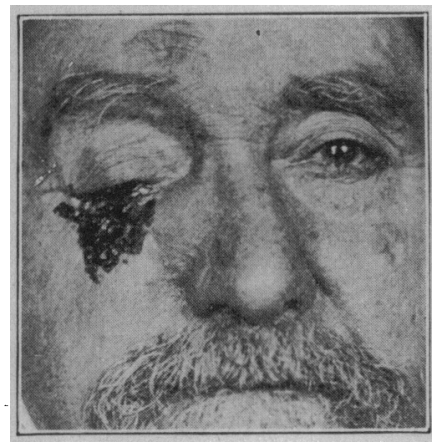

Fig. 8. - Epithelioma of lower id, inner and outer canthus, an malar region.

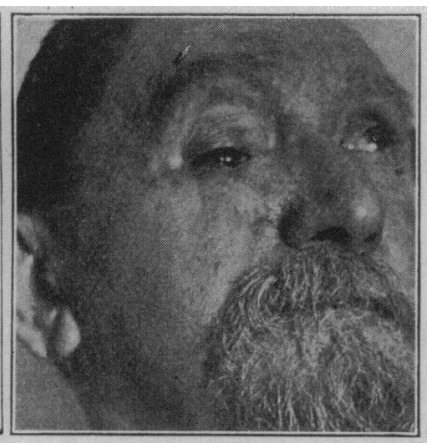

Fig. 9.-Result of three desiccation treatments; no recurrence in fifteen months.
5. There is less inflammatory reaction and discomfort to the patient.

6. There is less danger of symblepharon as a secondary complication.

Advantages over electrolysis or zinc mercury ionization are :

1. Desiccation destroys, from without inward, with an even distribution of destructive action.

2. It is more rapid in action and less painful.

3. There is no danger of dizziness or syncope.

4. Healing is more rapid.

It is not to be understood that the foregoing methods are always to be condemned, for in competent hands excellent results have been obtained by them all; but experience has taught me that desiccation possesses greater advantages, compared point for point, than any of these methods for the treatment of the lesions under discussion.

\section{RESULTS IN LOCALIZED EPITHELIOMAS}

Of the eighty-four cases of localized epitheliomas, the average age of the patients was 52 years. The youngest was 28 years. Biopsies were made in twenty-one cases, and these all proved to be of the basal cell type. In the remaining sixty-three cases, the clinical diagnosis was clear; hence a pathologic examination did not seem necessary or prudent, because a patient is more or less jeopardized from a curative and cosmetic standpoint by incising the growth and would to a certain measure defeat the object of desiccation. A biopsy, therefore, was performed only in such cases as seemed doubtful.

Of the cases treated, one has not recurred in six years, two in five years, two in four years, five in three years, seventeen in two years, and twenty-seven in one year. Fifteen have not recurred in periods ranging from three to eleven months. Those under one year will not be considered in computing percentage. There were six recurrences within one year and four within two years. Of these ten cases that did recur, six patients have remained well one year or

more after a second treatment. Two cases recurred a second time within a year, but the patients have remained well for a year or more after a third treatment. Two recurred a third time within one year; one of these patients is apparently well after six months, and the other is under treatment at the present time. Two of the remaining five patients died within a year, and at time of death there was no recurrence. The remaining three I have not up to the present time been able to follow. Deducting the fifteen cases in which less than a year has elapsed since treatment, the two patients who died and the three cases unaccounted for, a percentage will be computed from sixty-four cases. Fifty-four have not recurred in from one to six years, which is 84.375 per cent. of cases in which there has been freedom from recurrence one year or over after one treatment. If the ultimate results of the cases that recurred and received a second or third treatment are taken into consideration, the percentage of apparent cures, using freedom from recurrences one year or over as a basis, will be raised to 96.875 per cent. It is possible that the three cases unaccounted for would raise the percentage even higher. With knowledge learned from mistakes and more thoroughness of technic gained from experience, and also with the success obtained with basal cell epitheliomas in other parts of the body, which is not recorded in this paper, I believe that practically all patients with basal cell epitheliomas can be permanently cured, or if in a small percentage there is a recurrence, they may be treated with at least an equal chance of cure as before the first treatment.

Eight cases of very advanced epitheliomas involving sinuses or orbit were treated by desiccation for palliative effect only. Six of these cases were recurrences after operative surgery, and continued radium or Roentgen-ray treatment. The other two had been treated by Roentgen rays only. At the time these patients applied for treatment, they were absolutely inoperable. Much was done to relieve pain and stay the progress of the disease. No cures were accomplished or expected in these cases.

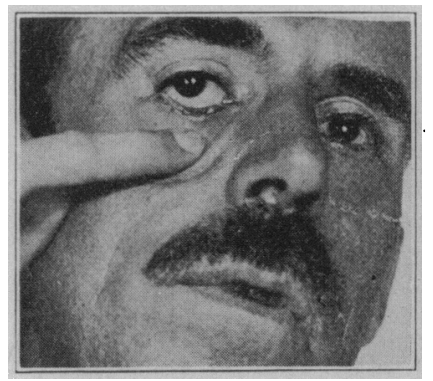

Fig. 10. - Epithelioma of lower lid and inner canthus, involving cartilage.

Two cases of advanced round cell sarcoma of the lids were treated, one on the upper right lid of a girl baby aged 8 months, that had recurred two weeks after excision. There was a diversity of opinion in this case as to the diagnosis. One pathologist reported it as a round cell sarcoma, while the other was doubtful. At any rate, there has been 'no recurrence eight months after one desiccation treatment with very slight deformity.

The other case was a round cell sarcoma of the lower right lid of a man aged 45 years. The diagnosis 
was confirmed by pathologic examination. This was also a recurrence two weeks after operation. One desiccation treatment cleared it up with practically no evidence of deformity other than absence of cilia. Nine months after treatment a growth the size of a pea reappeared at the inner canthus. This was treated by desiccation three months ago, and it is too early to report the ultimate result.

Four cases of melanosarcoma were treated. Two of these involved the palpebral and bulbar conjunctiva, and consisted of several slightly elevated pigmented growths showing a tendency to merge. In one, opera-

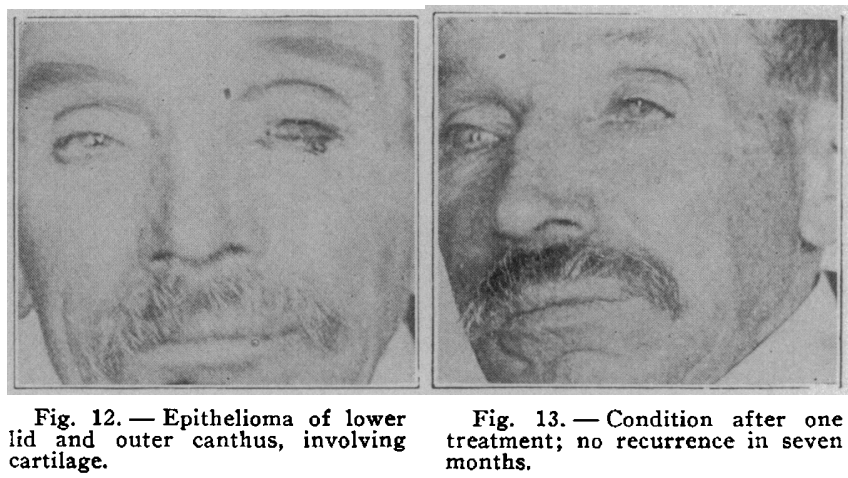

tion had been performed with recurrence. In one case there was no recurrence in ten months, but in the other there is a slight reappearance of pigmentation in one small area, and will be treated again. The other two cases were very much alike and consisted of elevated pigmented growths about three-eighths inch in diameter, involving the bulbar conjunctiva and cornea. The diagnosis was confirmed by pathologic examination. These cases were treated in the same week. There has been no recurrence in either case in four months.

$$
\text { BENIGN LESIONS }
$$

Lesions such as warts, moles with flat or pedunculated bases and xanthoma, even though they are on the margin of the lid and involving the palpebral conjunctiva, are easily removed with one desiccation treatment without noticeable scarring. There have been no failures in a total of sixty-three cases treated.

Erectile cutaneous or subcutaneous angiomas also respond to one application, if the desiccation is carried deeply enough to obliterate the blood lakes, and a good cosmetic result is the rule. There were two unsatisfactory results in eleven cases, the cause of which was failure to desiccate deeply enough.

Three cases of lupus erythematosus of the lids responded to one desiccation treatment.

Five cases of chronic granular conjunctivitis were each very much improved by one desiccation treatment. An ophthalmologist who observed one result said that as much had been accomplished by this treatment as could be accomplished in three months with silver nitrate.

Two cases of pterygium complicating epitheliomas of the inner canthi were successfully treated by desiccation. Another case, in which there were eight recurrences after operation, was treated by this method, but sufficient time has not yet elapsed to tell whether or not there will be a recurrence. Slight symblepharon resulted in this case.

Three cases of indolent corneal ulcer that had resisted ordinary remedial measurés responded to one desiccation treatment.

\section{CONCLUSIONS}

1. Desiccation is a successful treatment for localized basal cell epitheliomas of the lids and canthi, both from a curative and a cosmetic standpoint.

2. In advanced epitheliomas of these regions when sinuses or orbit are involved, complete success is not certain because of the inaccessibility of the diseased tissue, and is applied for palliative reasons, when operation fails or is contraindicated.

3 . The results thus far in round cell and melanosarcoma of the lids and conjunctiva have been good, but a sufficient time has not elapsed in any case to determine ultimate results.

4. Success is assured in benign growths of the lids, such as angiomas, warts, moles, xanthoma and lupus erythematosus.

5. Desiccation may be used to advantage for the treatment of pterygium.

6. The method is valuable in the treatment of granular conjunctivitis and corneal ulcers.

7. Symblepharon usually does not follow desiccation.

8. There is no danger of applying the desiccation treatment to growths on the cornea, as it is under perfect control.

NoTE.-Other photographs of epithelioma cases clear enough for publication, showing freedom from recurrence for over one year, have been published before, and hence are not available at this time. For this reason, only comparatively recent pictures can be shown.

1809 Chestnut Street.

\section{ABSTRACT OF DISCUSSION}

Dr. S. LewIS Ziegler, Philadelphia: High frequency desiccation for the removal of accessible growths is applicable whether the new growth is malignant or benign. The advantages which this procedure will present to ophthalmic surgeons are (1) the simplicity of the technic; (2) the rapid healing without scar tissue; (3) the low percentage of recurrence. The disadvantages are (1) the elaborate and costly apparatus necessary to obtain the best results, and (2) the danger of too deep destruction of tissue unless the operator is skilful and thoroughly trained in this special field of work. Dr. Clark recently treated for me a case of angioid growth on the forehead, the result of traumatism many years ago,

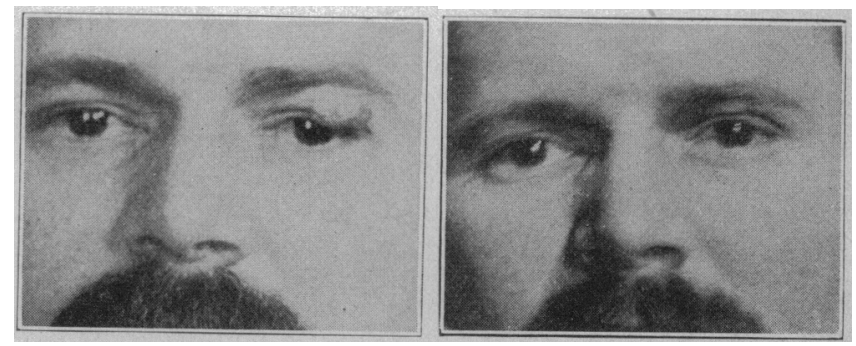

Fig. 14.-Angioma of upper lid. Fig. 15.-Result of one treatment.

but which had recently begun to show malignant characteristics. The result following a single treatment has been very satisfactory, and scarcely a mark is now visible. The possibility of removing growths from the cornea and sclerocorneal limbus is of great interest to us. This of course requires very careful technic. The rugae and granulomas of chronic trachoma will often yield to this technic and thus save us more extensive operative interference. I hope soon to try its efficacy in a case of keloid following a traumatic pterygium. It is also possible that a method may be devised of introducing the needle into the lacrimal sac and of so regenerating its lining that excision will not be necessary. I have no doubt that many other uses will suggest themselves as we continue to study the possibilities of this procedure. I am especially interested in the fact that the technic 
of this procedure shows the advantages of the principle of high voltage and low amperage, a principle that $I$ have long since advocated in the application of the galvanic current to the eye; and also that it tends to conserve tissue and thus yield a good cosmetic result, which requirement was the aim of my galvano-cautery operation for ectropion and entropion.

DR. W. C. Posey, Philadelphia: I have followed a number of the cases shown and Dr. Clark has treated others for me. In a number of cases, xanthoma cleared away after one application of the method, leaving absolutely no scar. Two other cases of diffuse sarcoma of the conjunctiva were cleared away. In the first case, one in which there had been a recurrence after primary operation on the growth some ten years previously, a second recurrence five years afterward was cleared away, I think, after two sittings. The other case, an isolated sarcoma of the conjunctiva of the lower lid, yielded to one sitting. A case of diffuse injury to the cornea, in which there was a diffuse haze of the epithelium and subepithelial tissues which persisted for several weeks after the use of dionin and yellow oxid, cleared away in forty-eight hours in large measure following one application of this method. The method is of very great value in neoplasms of the lids, obviating doing extensive plastic operations and avoiding the resulting scar.

Dr. W. L. Clark, Philadelphia: One point I probably did not make clear. In treating the cornea I do not use a stream of sparks. When a stream of sparks is used through a spark gap the action is quite superficial. It should never be used in a stream on the cornea. The needle is just allowed to brush the tissue, the merest, faintest contact. If this were not done it would be impossible to concentrate it on a certain point. There is some spreading in fan shape. You must have the needle in direct contact. It is not necessary to have two poles; just one.

\section{BISMUTH PASTE IN CHRONIC SUPPU- RATIVE SINUSES AND EMPYEMA}

INCORRECT TECHNIC AS A CAUSE OF FAILURE IN ITS APPLICATION*

EMIL G. BECK, M.D.

Feliow of the American College of Surgeons; Surgeon to the North Chicago Hospital

CHICAGO

The prevalence of suppurative sinuses has greatly diminished during the past decade. This is due partly to our ability to prevent their formation by treating cold abscesses and joint diseases more scientifically, and partly to the employment of the newer and more effective methods of treatment of sinuses when they already exist.

The present war.in Europe, however, will result in a tremendous increase in this class of cases. I am informed that Dr. Alexis Carrel, from one of the field hospitals of France, that nearly every case of gunshot wound is infected before it reaches the hospital. Dr. Carrel further reports that these gunshot and shrapnel wounds are followed by suppurative sinuses, and that there are thousands of such cases in France. No doubt, the other belligerent countries are having the same experience, so that after the war is over there will remain for years an army of invalids with these chronic suppurations.

It is principally for this reason that I am tempted again to present the subject of treating this class of cases.

Ten years have elapsed since the bismuth treatment of suppurative sinuses and abscess cavities was intro-

* Read before the Section on Surgery, General and Abdominal, at the Sixty-Seventh Annual Session of the American Medical Association,
Detroit, June, 1916. duced into surgery. During these years this method of treatment has been given a most thorough and impartial trial. When first introduced, surgeons in this country and abroad readily took up the method, because it appealed to them as a rational treatment, and because my report contained such surprisingly successful results. Besides, the material at hand was plentiful, and patients were willing to submit to any form of operative or nonoperative treatment which gave the slightest prospect for a cure.

Shortly afterward there came reports from the various clinics. Some surgeons showed far better results than my own, others less favorable results, and in the hands of a few the procedure was more or less a failure. Undoubtedly these reports represent only a fraction of the cases in which the treatment was employed, for many country practitioners who used the bismuth treatment did not report their results. The accompanying table presents the statistics recorded in the literature.

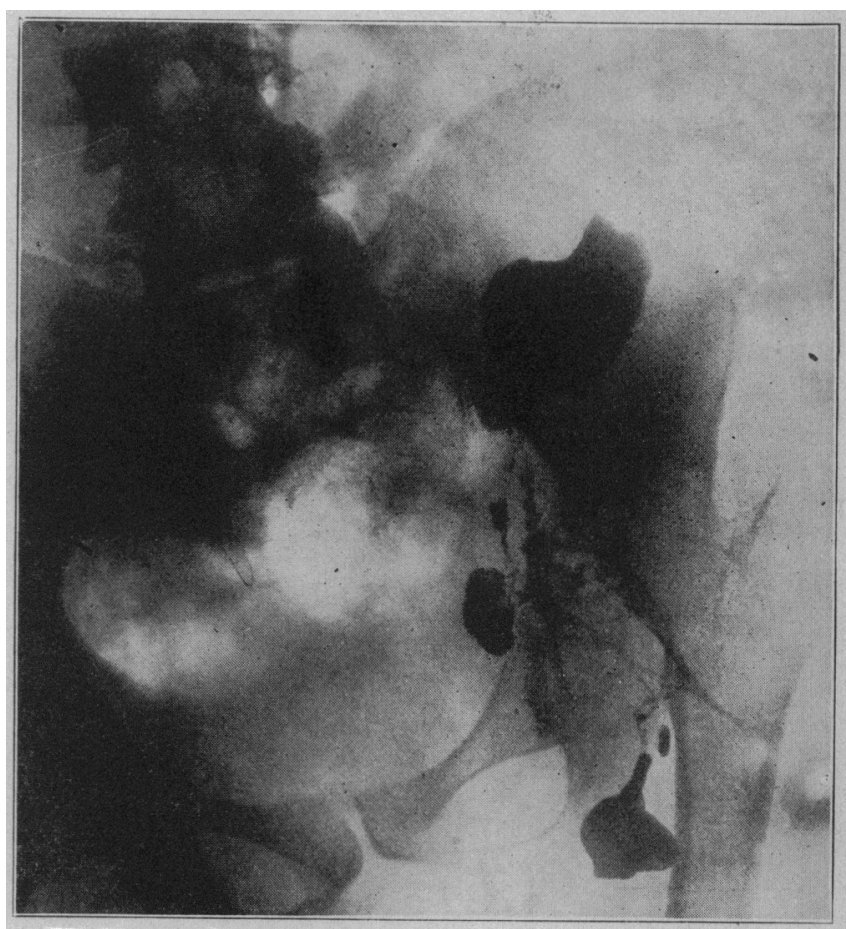

Fig. 1.-Injection of sinuses from tuberculous hip, showing narrowing of channels of preexisting abscess cavities.

This series of 527 cases ( 319 nose and throat cases excluded), from so wide a range of sources, gives an average of 80.64 per cent. of cures, the lowest being 12.5 per cent., and the highest, 100 per cent.

How can we explain the lack of uniformity in results? To ascertain this I have made observations in clinics here and abroad, I have conferred and corresponded with surgeons, and finally I have come to the conclusion that faulty technic is the chief cause of failures.

At the North Chicago Hospital, where the method was started in 1906, we have had exceptional opportunities for treating suppurative sinuses and abscesses with this method, the material now amounting to over 1,800 cases, which includes almost every variety of suppurative disease. No doubt, we committed many blunders before our technic was perfected; but I feel that with accumulated experience and from what I have learned through my own mistakes and those of others, I am now in a position to outline for the pro- 